\title{
Identification of DIR1-Dependant Cellular Responses in Guard Cell Systemic Acquired Resistance
}

\author{
Lisa David ${ }^{1,2}$, Jianing Kang ${ }^{1,2,3}$, Josh Nicklay ${ }^{4}$, Craig Dufresne ${ }^{5}$ and Sixue Chen ${ }^{1,2,6,7 *}$ \\ ${ }^{1}$ Department of Biology, University of Florida, Gainesville, FL, United States, ${ }^{2}$ University of Florida Genetics Institute (UFGl), \\ Gainesville, FL, United States, ${ }^{3}$ College of Life Science, Northeast Agricultural University, Harbin, China, ${ }^{4}$ Thermo Fisher Scientific, \\ Somerset, NJ, United States, ${ }^{5}$ Thermo Training Institute, Thermo Fisher Scientific, West Palm Beach, FL, United States, ${ }^{6}$ Plant \\ Molecular and Cellular Biology Program, University of Florida, Gainesville, FL, United States, ${ }^{7}$ Proteomics and Mass \\ Spectrometry, Interdisciplinary Center for Biotechnology Research (ICBR), University of Florida, Gainesville, FL, United States
}

OPEN ACCESS

Edited by:

Robert David Hall,

Wageningen University and Research,

Netherlands

Reviewed by:

Robin K. Cameron,

McMaster University, Canada

Zhentian Lei,

University of Missouri, United States

*Correspondence:

Sixue Chen

schen@ufl.edu

Specialty section:

This article was submitted to

Metabolomics,

a section of the journal

Frontiers in Molecular Biosciences

Received: 24 July 2021 Accepted: 15 November 2021

Published: 17 December 2021

Citation:

David L, Kang J, Nicklay J, Dufresne C and Chen S (2021) Identification of DIR1-Dependant Cellular Responses

in Guard Cell Systemic

Acquired Resistance.

Front. Mol. Biosci. 8:746523. doi: 10.3389/fmolb.2021.746523
After localized invasion by bacterial pathogens, systemic acquired resistance (SAR) is induced in uninfected plant tissues, resulting in enhanced defense against a broad range of pathogens. Although SAR requires mobilization of signaling molecules via the plant vasculature, the specific molecular mechanisms remain elusive. The lipid transfer protein defective in induced resistance 1 (DIR1) was identified in Arabidopsis thaliana by screening for mutants that were defective in SAR. Here, we demonstrate that stomatal response to pathogens is altered in systemic leaves by SAR, and this guard cell SAR defense requires DIR1. Using a multi-omics approach, we have determined potential SAR signaling mechanisms specific for guard cells in systemic leaves by profiling metabolite, lipid, and protein differences between guard cells in the wild type and dir1-1 mutant during SAR. We identified two long-chain $18 \mathrm{C}$ and $22 \mathrm{C}$ fatty acids and two $16 \mathrm{C}$ wax esters as putative SAR-related molecules dependent on DIR1. Proteins and metabolites related to amino acid biosynthesis and response to stimulus were also changed in guard cells of dir11 compared to the wild type. Identification of guard cell-specific SAR-related molecules may lead to new avenues of genetic modification/molecular breeding for disease-resistant plants.

Keywords: Arabidopsis thaliana, systemic acquired resistance, multi-omics, proteomics, metabolomics, lipidomics

\section{INTRODUCTION}

Since the dawn of agriculture, epidemics of plant pathogens have caused devastating impacts to food production. The plant bacterial pathogen Pseudomonas syringae (including more than sixty known host-specific pathovars) infects broad-ranging and agriculturally relevant plants (Saint-Vincent et al., 2020). Although it was first isolated from lilac (Syringa vulgaris) in 1899, strains of P. syringae are found in many important crops, including beans, peas, tomatoes, and rice (Saint-Vincent et al., 2020). P. syringae pv tomato (Pst) is a pervasive phytopathogenic bacterium that causes damage to a wide range of host crop species. It has been a useful model pathogen for studying host immune response since the sequencing and annotation of the 6,397,126-bp genome and two plasmids which was funded by the NSF Plant Genome Research Program (Hirano and Upper, 2000). Pst infects leaves for chemical nutrients such as carbohydrates, amino acids, organic acids, and ions that are leaked to the leaf apoplast during phloem loading/unloading (Hirano and Upper, 2000). Pst causes bacterial brown spot disease in fruit and leaves, damaging crop plants. However, more devastating 
than brown spot is the unique ability of Pst to nucleate supercooled water to form ice. In the species of $P$. syringe exhibiting the ice nucleation phenotype, ice-nucleation proteins on the outer membranes of bacterial membranes form aggregates that arrange water into arrays and promote phase change from liquid to solid. The frost-sensitive plants are injured when ice forms in leaf tissues at subzero temperature (Hirano and Upper, 2000). Pst has been used extensively to study pathogen infection in numerous host plants including tomato and Arabidopsis. The latter is a reference dicot species with a short life cycle, fully sequenced genome, and rich genetic resources, providing an ideal system to understand how plants may be modified to improve their defense and productivity.

Systemic acquired resistance (SAR) is a long-distance plant immune response that improves the immunity of distant tissues after local exposure to a pathogen (Chester, 1933; Ross, 1966; Shah and Zeier, 2013; David et al., 2019). Mobile SAR signaling molecules reach distal tissues from pathogen-infected tissues via the plant vasculature and are perceived by cells in the systemic tissues to initiate the global SAR response (Conrath, 2006; Chanda et al., 2011). Perception of mobilized SAR signals in systemic tissue activates cellular defense responses leading to a "primed" condition in systemic, noninfected tissues. Priming enables the plant to maintain a vigilant or alarmed status by which they react faster and more effectively to pathogen attack (Conrath, 2011; Misra and Chaturvedi, 2015). Stomatal pores on leaf surfaces formed by pairs of guard cells are common entry sites for pathogenic bacteria. The specialized guard cells control the opening and closure of stomatal pores in response to environmental conditions (Melotto et al., 2008). When stomatal guard cells recognize Pst via pattern recognition receptors, stomata close within $1-2 \mathrm{~h}$ and reopen after $3 \mathrm{~h}$. Reopening is due to a phytotoxin produced by some strains of $P$. syringae called coronatine (COR), which structurally mimics the active form of the plant hormone jasmonic acid-isoleucine (Melotto et al., 2008). As a primary entry site for bacteria into the plant tissue, the stomata are at the frontline in plant immune defense (Zhu et al., 2012). Our previous research showed that systemic leaves of Pst-primed wild-type (WT) Arabidopsis have smaller stomata apertures in distant leaves than mock-primed plants, and Pst does not widen stomata aperture in Pst-primed leaves, as it does in mock-primed plants (David et al., 2020). Reduced stomatal aperture of Pst-primed plants associated with reduced bacterial entry into the leaf apoplastic space and reduced bacterial proliferation (David et al., 2020).

Using a 3-in-1 extraction method to obtain proteins, metabolites, and lipids from the same guard cell samples, we conducted multi-omics to identify SAR-related components in guard cells of WT Arabidopsis and a T-DNA insertional mutant of defective in induced resistance 1 (DIR1). DIR1 encodes a putative apoplastic lipid transfer protein involved in SAR. Arabidopsis plants with mutations in DIR1 exhibit WT-level local resistance to avirulent and virulent Pst, but pathogenesisrelated gene expression is abolished in uninoculated distant leaves, and mutants fail to develop SAR (Maldonado et al., 2002). Champigny et al. (2013) examined the presence of
DIR1 in petiole exudates from SAR-induced Arabidopsis leaves that were injected with Pst. The exudates from the Pstinjected leaves showed the presence of DIR1 beginning at $30 \mathrm{~h}$ post-infection (hpi) and peaking at $\sim 45$ hpi (Champigny et al., 2013). Interestingly, the small 7-kD DIR1 protein was also detected in dimeric form in the petiole exudates (Champigny et al., 2013). DIR1 is conserved in other land plants including tobacco and cucumber, and several identified SAR signals are dependent on DIR1 for long-distance movement, e.g., dehydroabietinal (DA) (Chaturvedi et al., 2012), azelaic acid (AzA) (Jung et al., 2009) and glycerol-3-phosphate (G3P) (Nandi et al., 2004; Adam et al., 2018). Although most of the LTPs have basic pIs, DIR1 has an acidic pI of 4.25. Martinière et al. (2018) found that the apoplastic environment has a more acidic $\mathrm{pH}$ than the cellular environment, ranging between 4.0 and 6.3 , so perhaps the acidic $\mathrm{pI}$ of DIR1 relates to its function in a more acidic environment where it may be neutral, similar to abscisic acid which is transported in the apoplast during stress response (Cornish and Zeevaart, 1985). However, to date there is no evidence that DIR1 is transported in the apoplast.

DIR1 is comprised of 77 amino acids, but despite having cystine residues characteristic with lipid transfer proteins (LTPs), it has a low sequence identity with the previously characterized LTP1 and LTP2 in Arabidopsis (Lascombe et al., 2006). Lascombe et al. (2008) used x-ray crystallography to compare the structures of DIR1 to LTP1 by examining their interactions with and without various lipid substrates, including lysophosphatidylcholines (LPCs) with various fatty acid chain lengths (LPC C14, LPC C16, and LPC C18). The results showed that DIR1 showed a greater affinity for LPCs with fatty acid chain lengths with $>14$ carbon atoms than LTP1. For the LPC with C18 fatty acid tails, the nonpolar $\mathrm{C} 18$ end was completely buried within the barrel structure of the DIR1 protein. DIR1 is unique among the LTPs due to its large internal cavity, capable of carrying two lipid molecules, and a proline-rich PxxPxxP motif (including Proline 24 to Proline 30). The Proline-rich regions of DIR1 may be involved in protein-protein interactions, as these regions are located at the surface of the protein and are fully accessible to the aqueous environment (Lascombe et al., 2008). These regions are putative candidates for docking of a protein signaling partner, or to other cell components. These features may lend themselves well to its role at a SAR-induced LTP because DIR1 is hypothesized to form a complex with azelaic acid-induced 1 (AZI1), localize to the endoplasmic reticulum and plasmodesmata (Champigny et al., 2011; Yu et al., 2013), and function as a carrier for neutral fatty acids in the apoplast. Many "box-like" LTPs, like DIR1, have a "lid"-like structure that encloses the lipid ligands inside the hydrophobic cavity during transport in the aqueous environment and have structural motifs that undergo conformational shifts to allow for lipid loading and unloading (Wong et al., 2019).

In this study, a multi-omics approach was employed to identify SAR signaling mechanisms in stomatal guard cells. The results show potential involvement of DIR1 in amino acid biosynthesis and carbon metabolism in guard cells during SAR. Importantly, four lipid components with long-chain fatty acids 
were identified as putative DIR1-related SAR signals in guard cells. Understanding molecular changes in guard cells during SAR response not only had led to new insights into the basic function of guard cells in the plant immune response but also may facilitate biotechnology and marker-based breeding for enhanced crop defense.

\section{MATERIALS AND METHODS}

\section{Plant Growth and Bacterial Culture}

A. thaliana WS (CS915) and dir1-1 (CS6389) seeds were obtained from the Arabidopsis Biological Research Center (Columbus, $\mathrm{OH}$, USA), and plants were grown as described in David et al. (2020). Briefly, seeds were vernalized at $4^{\circ} \mathrm{C}$ for 2 days before planting in soil and grown in controlled environmental chambers in a short-day (8-h light/16-h dark) environment with temperatures at $22^{\circ} \mathrm{C}$ under light and $20^{\circ} \mathrm{C}$ in the dark, a lighting set at $140 \mu \mathrm{mol} \mathrm{m} \mathrm{m}^{-2} \mathrm{~s}^{-1}$, and a relative humidity of $60 \%$. Two-week-old seedlings were transferred into individual pots and gown until mature rosette (stage 3.9) was observed at 5 weeks of age. Pseudomonas syringae pv. tomato DC3000 (Pst), the model pathogen for Arabidopsis SAR induction, was used for all the experiments and was cultured on agar media plates, made using autoclaved King's B media, and antibiotics rifampicin $(25 \mathrm{mg} / \mathrm{l})$ and kanamycin $(50 \mathrm{mg} / \mathrm{l})$ were added once the solution is cooled. After overnight incubation at $28^{\circ} \mathrm{C}$, Pst colonies were grown in King's B media without agar in solution overnight, pelleted by centrifugation at $6,000 \mathrm{~g}$ for $10 \mathrm{~min}$, and used for treatment of Arabidopsis plants.

\section{Stomata Aperture Measurements}

Inoculations and stomata aperture measurements were performed as described in David et al. (2020). Briefly, one fully expanded rosette leaf was given a primary inoculation via a needleless syringe, with Pst DC3000 $\left(\mathrm{OD}_{600}=0.02\right)$ suspended in $10 \mathrm{mM} \mathrm{MgCl}_{2}$. This plant is called of Pst-primed. At the same time, another plant was similarly inoculated with $10 \mathrm{mM} \mathrm{MgCl}$ only, and it is called mock-primed (Supplementary Figure S1). This mock and Pst-priming experiment was repeated three times. At 3 days postinoculation, one mature rosette leaf opposite to the injected leaf was detached from each plant and floated either in $10 \mathrm{mM} \mathrm{MgCl}_{2}$ or in Pst DC3000 $\left(\mathrm{OD}_{600}=0.2\right.$, in $\left.10 \mathrm{mM} \mathrm{MgCl}_{2}\right)$ in small petri dishes for 0,1 , or $3 \mathrm{~h}$ of secondary treatment in the growth chamber under the light conditions. A total of 150 stomata were measured for each treatment by collecting measurements of 50 stomata from three leaves taken from three individual plants for each treatment, and then the entire experiment was replicated three times. The leaves were collected and peeled using a clear tape; the peel from the abaxial side of the leaf was then placed on a microscope slide and imaged using a DM6000B light microscope (Leica, Buffalo Grove, IL USA) at 01 and $3 \mathrm{~h}$ post-secondary treatment. The stomatal apertures were analyzed using ImageJ software (National Institutes of Health, Bethesda, MD, USA; http://imagej.nih.gov/ij/). Two-way ANOVA and unpaired Student's t-test were conducted. The $p$-values less than 0.05 were considered statistically significant.
The data were plotted as mean with $95 \%$ confidence interval. Statistically significant different groups were marked by different letters.

\section{Pst DC3000 Entry and Growth Assays}

Pst entry assays were used to measure how much bacteria entered the apoplast after $3 \mathrm{~h}$ of Pst exposure. The 5-week-old plants were Pst-primed and mock-primed as described in the previous section. Three days after the primary inoculation, one uninfected leaf opposite to the one infected was detached and floated in Pst $\left(\mathrm{OD}_{600}=0.2\right)$ solution for $3 \mathrm{~h}$, then washed by vortexing in sterile water containing $0.02 \%$ Silwet (Su et al., 2017) and dried with sterile Kim wipes. In the aseptic environment of the laminar flow hood, an autoclaved hole puncher was used to obtain one $0.5-\mathrm{cm}$ disk from each leaf. Leaf disks were ground with a sterilized plastic tip in $100 \mu \mathrm{l}$ sterile $\mathrm{H}_{2} \mathrm{O}$ followed by a 1 : 1,000 serial dilution in sterile $\mathrm{H}_{2} \mathrm{O}$ for plating. A volume of $100 \mu \mathrm{l}$ from each dilution was plated on agar media containing rifampicin $(25 \mathrm{mg} / \mathrm{l})$ and kanamycin $(50 \mathrm{mg} / \mathrm{l})$. Colonies were counted after 2 days of incubation at $28^{\circ} \mathrm{C}$. The entire experiment was replicated three times using one leaf from three individual plants each time for a total of nine biological replicates from three independent experiments. The bacterial counts of nine replicates were used to calculate mean and 95\% confidence interval. The statistical analysis was done using 2-way ANOVA and unpaired t-test.

The Pst growth experiment determines how much bacteria grow in the apoplast after 3 days. As described above in the Pst entry experiment, nine independent replicates of 5-week-old Pstprimed and mock-primed plants were prepared, and 3 days after primary inoculation of one rosette leaf, all other uninfected rosette leaves were sprayed with Pst DC3000 $\left(\mathrm{OD}_{600}=0.2\right)$ and placed under a dome for top for $24 \mathrm{~h}$ to maintain humidity. After $24 \mathrm{~h}$, the dome was removed, and the infected plants stayed in the growth chamber for an additional $48 \mathrm{~h}$. One opposite leaf of each plant was then detached and washed in $0.02 \%$ Silwet, and one disk was taken from the leaf to make a 1 : 1,000 serial dilution and plated on media. Colonies were counted to determine how much bacteria were able to grow in the apoplast. The experiment was repeated three times with three sets of nine plants, and bacterial counts were used to calculate the mean and 95\% confidence interval. Statistical analysis was done using 2-way ANOVA and unpaired t-test. Statistically significant different groups were marked by different letters.

\section{Isolation of Enriched Guard Cells for Multi-Omics Experiments}

Enriched guard cell samples were prepared as described in David et al. (2021). Briefly, for each sample 144 mature leaves were collected. After removing the midvein with a scalpel, the leaves were blended for $1 \mathrm{~min}$ in a high-speed blender with $250 \mathrm{ml}$ of deionized water and ice. The sample was then filtered through a $200-\mu \mathrm{m}$ mesh filter. This process was repeated 3 times to obtain intact stomatal guard cells, which were collected immediately into $15-\mathrm{ml}$ Falcon tubes, snap frozen in liquid nitrogen, and stored in $-80^{\circ} \mathrm{C}$. Guard cell viability and purity were verified by staining 
with fluorescein diacetate and neutral red dye, which showed that guard cells remained intact and viable. Purity of the guard cell preparation has been verified by transcript abundances of six guard cell marker proteins and chlorophyll contents (Zhu et al., 2016).

\section{3-In-1 Extraction of Proteins, Metabolites and Lipids From Guard Cell Samples}

We adapted a protocol to simultaneously extract metabolites, lipids, and proteins from a single whole leaf or guard cell sample (Kang et al., 2021). Briefly, a chloroform and methanol solution is added to samples that are in an aqueous isopropanol solution. This process induces the formation of two solvent layers-an upper aqueous phase containing hydrophilic metabolites and a lower organic phase containing lipids and other hydrophobic metabolites. The proteins are at the interphase. Components were normalized from internal standards that were added during the first step of extraction. Internal standards included, for proteins, $60 \mathrm{fmol}$ digested bovine serum albumin (BSA) peptides per $1 \mu \mathrm{g}$ sample protein; for metabolites, $10 \mu \mathrm{l} 0.1 \mathrm{nmol} / \mu \mathrm{l}$ lidocaine, and camphorsulfonic acid; and for lipids, $10 \mu \mathrm{l} 0.2 \mu \mathrm{g} / \mu \mathrm{l}$ deuterium labeled 15:0-18:1(d7) phosphatidylethanolamine (PE) and 15: 0-18:1(d7) diacylglycerol (DG). The lipid extracts were dried under nitrogen gas to prevent oxidation and stored in $-80^{\circ} \mathrm{C}$. The lipid extract was later dissolved in $1 \mathrm{ml}$ isopropanol for LC-MS/ MS analysis. Aqueous metabolites were lyophilized and placed at $-80^{\circ} \mathrm{C}$, and pellets were later solubilized in $100 \mu \mathrm{l} 0.1 \%$ formic acid for LC-MS/MS analysis. Protein was precipitated in cold $80 \%$ acetone at $-20^{\circ} \mathrm{C}$ overnight, followed by removal of acetone using glass pipettes, and then protein samples were dried in a speedvac.

\section{Protein Digestion and LC-MS/MS}

Four biological replicates of mock-primed and Pst-primed guard cell samples from WT and dir1-1 genotypes were prepared for proteomic experiments. Protein samples were resuspended in $50 \mathrm{mM}$ ammonium bicarbonate, reduced using $10 \mathrm{mM}$ dithiothreitol (DTT) at $22^{\circ} \mathrm{C}$ for $1 \mathrm{~h}$, and alkylated with $55 \mathrm{mM}$ chloroacetamide in the darkness for $1 \mathrm{~h}$. Trypsin (Promega, Fitchburg, WI, USA) was added for digestion (enzyme: sample $=1: 100, \mathrm{w} / \mathrm{w}$ ) at $37^{\circ} \mathrm{C}$ for $16 \mathrm{~h}$. The digested peptides were desalted using a micro ZipTip minireverse phase (Millipore, Bedford, MA, USA) and then lyophilized to dryness. The peptides were resuspended in $0.1 \%$ formic acid for mass spectrometric analysis.

The bottom-up proteomics data acquisition was performed on an EASY-nLC 1200 ultraperformance liquid chromatography connected to an Orbitrap Exploris 480 with a FAIMS Pro instrument (Thermo Scientific, San Jose, CA, USA). The peptide samples were loaded in $5-\mu \mathrm{l}$ injections to an IonOpticks Aurora $0.075 \times 250 \mathrm{~mm}, 1.6-\mu \mathrm{m} 120-\AA$ analytical column, and the column temperature was set to $50^{\circ} \mathrm{C}$ with a sonation oven. The flow rate was set at $400 \mathrm{nl} / \mathrm{min}$ with solvent $\mathrm{A}$ $(0.1 \%$ formic acid in water $)$ and solvent $\mathrm{B}(0.1 \%$ formic acid and $80 \%$ acetonitrile) as the mobile phases. Separation was conducted using the following gradient: $3-19 \%$ B in $108 \mathrm{~min} ; 19-29 \%$ B in $42 \mathrm{~min} ; 29-41 \% \mathrm{~B}$ in $30 \mathrm{~min}$. The full MS1 scan (m/z 350-1,200) was performed on the Orbitrap Exploris with a resolution of 120,000 . The FAIMS voltages were on with a FAIMS CV (V) set at -50 . The RF lens (\%) was set to 40, and a custom automatic gain control (AGC) target was set with a normalized AGC target (\%) set at 300. Monoisotopic precursor selection (MIPS) was enforced to filter for peptides with relaxed restrictions when too few precursors are found. Peptides bearing two to six positive charges were selected with an intensity threshold of $5 \mathrm{e} 3$. A custom dynamic exclusion mode was used with a 60-s exclusion duration, and isotopes were excluded. Datadependent MS/MS was carried out with a three FAIMS CV loop $(-50,-65,-80)$. The MS/MS Orbitrap resolving power was set to 60,000 with a $2-\mathrm{m} / \mathrm{z}$ quadrupole isolation. The top speed for data-dependent acquisition within a cycle was set to $118 \mathrm{~m}$ of maximum injection time. The MS/MS mass tolerance was set to $10 \mathrm{ppm}$. Fragmentation of the selected peptides by higher-energy collision dissociation (HCD) was done at $30 \%$ of normalized collision energy and a $2-\mathrm{m} / \mathrm{z}$ isolation window. The MS2 spectra were detected by defining first the mass scan range as $120 \mathrm{~m} / \mathrm{z}$ and the maximum injection time as $118 \mathrm{~m}$.

\section{Metabolite and Lipid LC-MS/MS}

The untargeted metabolomic approach used the high-resolution Orbitrap Fusion Tribrid mass spectrometer (Thermo Fisher Scientific, Waltham, MA, USA) with Vanquish $^{\mathrm{TM}}$ UHPLC liquid chromatography and is described in detail in David et al. (2020). An Accucore C18 $(100 \times 2.1 \mathrm{~mm}$, particle size $2.6 \mu \mathrm{m})$ column was used for metabolites with solvent A $(0.1 \%$ formic acid in water) and solution $\mathrm{B}(0.1 \%$ formic acid in acetonitrile). The column chamber temperature was to $55^{\circ} \mathrm{C}$. The pump flow rate was set to $0.45 \mathrm{ml} / \mathrm{min}$. The LC gradient was set to 0 min: $1 \%$ of solvent B (i.e., $99 \%$ of solvent A), 5 min: $1 \%$ of B, 6 min: $40 \%$ of B, 7.5 min: $98 \%$ of B, $8.5 \mathrm{~min}$ : $98 \%$ of $\mathrm{B}$, 9 min: $0.1 \%$ of $B, 10$ min stop run. To enhance identification, an Acquire X MSn data acquisition strategy was used which employs replicate injections for exhaustive sample interrogation and increases the number of identified compounds in the sample with distinguishable fragmentation spectra. Electrospray ionization (ESI) was used in both positive and negative modes with a spray voltage for positive ions $(\mathrm{V})=3,500$ and a spray voltage for negative ions $(V)=2,500$. Sheath gas was set to 50 , auxiliary gas was set at 1 , and sweep gas was set to 1 . The ion transfer tube temperature was set at $325^{\circ} \mathrm{C}$, and the vaporizer temperature was set at $350^{\circ} \mathrm{C}$. Full MS1 used the Orbitrap mass analyzer (Thermo Fisher Scientific, Waltham, MA, USA) with a resolution of 120,000 , scan range $(\mathrm{m} / \mathrm{z})$ of $55-550$, MIT of 50 , AGC target of $2 \mathrm{e} 5$, one microscan, and RF lens set to $50 \%$. For untargeted lipidomics, a Vanquish HPLC-Q Exactive Plus system was used with an Acclaim C30 column $(2.1 \mathrm{~mm} \times 150 \mathrm{~mm}$, $3 \mu \mathrm{m})$. Solution A for lipids consisted of $0.1 \%$ formic acid, $10 \mathrm{mM}$ ammonium formate, and $60 \%$ acetonitrile. Solution B for lipids consisted of $0.1 \%$ formic acid, $10 \mathrm{mM}$ ammonium formate, and 90:10 acetonitrile: isopropyl alcohol. The column chamber temperature was set to $40^{\circ} \mathrm{C}$. The pump flow rate was set to $0.40 \mathrm{ml} / \mathrm{min}$. The LC gradient was set to $0 \mathrm{~min}: 32 \%$ of solvent $\mathrm{B}$ (i.e., $68 \%$ of solvent A), $1.5 \mathrm{~min}: 45 \%$ of B, $5 \mathrm{~min}: 52 \%$ of B, 8 min: $58 \%$ of B, 11 min: $66 \%$ of B, 14 min: $70 \%$ of B, 18 min: $75 \%$ of B, 
$21 \mathrm{~min}: 97 \%$ of B, $26 \mathrm{~min}: 32 \%$ of B, 32 min stop run. The method for the Q Exactive Plus mass spectrometer included a 32-min duration time, 10-s chromatogram peak width with full MS, and ddMS2. Ion fragmentation was induced by HCD, with positive and negative polarity switching and a default charge state of 1 . Full MS1 used the Orbitrap mass analyzer with a resolution of 70,000 , one microscan, an AGC target set to $1 \mathrm{e} 6$, and a scan range from 200 to $2,000 \mathrm{~m} / \mathrm{z}$. The ddMS2 scan used one microscan, resolution of 35,000, AGC target 5e5, MIT of $46 \mathrm{~m}$, loop count of 3 , isolation window of $1.3 \mathrm{~m} / \mathrm{z}$, and a scan range of $200-2,000 \mathrm{~m} / \mathrm{z}$ for positive and negative polarity.

\section{Data Analysis for Proteins, Metabolites, and Lipids}

For LC-MS/MS proteomic data analysis, we used Proteome Discoverer $^{\mathrm{TM}} 2.4$ (Thermo Fisher Scientific, Waltham, MA, USA) with the search engine SEQUEST algorithm to process raw MS files. Spectra were searched using the TAIR10 protein database with the following parameters: $10 \mathrm{ppm}$ mass tolerance for MS1 and $0.02 \mathrm{da}$ as mass tolerance for MS2, two maximum missed tryptic cleavage sites, a fixed modification of carbamidomethylation $(+57.021)$ on cysteine residues, dynamic modifications of oxidation of methionine (+15.996), and phosphorylation $(+79.966)$ on tyrosine, serine, and threonine. Search results were filtered at $1 \%$ false discovery rate (FDR), and the peptide confidence level was set for at least two unique peptides per protein for protein identification. Relative protein abundance in Pst-primed and control dir1-1 and WS guard cell samples was measured using label-free quantification in Proteome Discoverer $^{\mathrm{TM}} 2.4$ (Thermo Scientific, Bremen, Germany). Proteins identified and quantified in all four out of four sample replicates were used. Peptides in mock-primed and Pst-primed samples were quantified as area under the chromatogram peak. Peak areas were normalized by total protein amount. The average intensity of four Pst-primed dir11 vs. four Pst-primed WS samples was compared as a ratio, and two criteria were used to identify significantly altered proteins: 1 ) increase or decrease of 2-fold (Pst-primed dir1-1/Pst-primed WS) and 2) $p$-value from an unpaired Student's t-test less than 0.05 . For untargeted metabolomics, Compound Discover ${ }^{\mathrm{TM}} 3.0$ Software (Thermo Scientific, Bremen, Germany) was used for data analyses. Raw files from four replicates of dir1-1 Pst-primed and four replicates of WS Pst-primed guard cells were used as input. Spectra were processed by aligning retention times. Detected compounds were grouped and gaps filled using the gap filling node in Compound Discover that fills in missing peaks or peaks below the detection threshold for subsequent statistical analysis. The peak area was refined from normalized areas while marking background compounds. Compound identification included predicting compositions, searching the mzCloud spectra database, and assigning compound annotations by searching ChemSpider; pathway mapping to KEGG pathways and to Metabolika pathways was included for functional analysis of the metabolites. The metabolites were scored by applying mzLogic, and the best score was kept. Peak areas were normalized by the positive and negative mode internal standards (lidocaine and camphorsulfonic acid, respectively) added during sample preparation. For untargeted lipidomics data analyses, Lipid Search $4.1^{\mathrm{TM}}$ and Compound Discover ${ }^{\mathrm{TM}}$ 3.0 (Thermo Scientific, Bremen, Germany) were used. Raw files from three replicates of mock-primed and three replicates of Pst-primed guard cells were uploaded Lipid Search $4.1^{\mathrm{TM}}$ for annotation of lipids found in all the samples. A mass list was generated for uploading to Compound Discover ${ }^{\mathrm{TM}}$ 3.0 Software. This mass list was used for compound identification along with predicted compositions, searching the mzCloud spectra database, and assigning compound annotations by searching ChemSpider. Peak areas were normalized by median-based normalization. For both metabolomics and lipidomics, the average areas of four dir11 Pst-primed vs. four WS Pst-primed metabolite samples were compared as a ratio and two criteria were used to determine significantly altered metabolites or lipids: 1) increase or decrease of 2-fold (dir1-1 Pst-primed/WS Pst-primed) and 2) $p$-value from an unpaired Student's t-test less than 0.05 .

\section{Accession Numbers and Data Repository Information}

The datasets presented in this study can be found in online repositories. The names of the repository/repositories and accession number(s) can be found as follows: all protein MS raw data and search results have been deposited to the ProteomeXchange Consortium via the PRIDE partner repository with the data set identifier PXD024991. All metabolite and lipid MS raw data and search results have been deposited to the MetaboLights data repository with the data set identifier MTBLS2614.

\section{RESULTS AND DISCUSSION}

The altered stomatal priming response in dir1-1 is associated with increased bacterial colonization. We have previously characterized that a smaller stomatal aperture in the distant leaves of Pst-primed WT Arabidopsis improves immunity by allowing fewer bacteria to enter apoplastic spaces (David et al., 2020). The experimental design is illustrated in Supplementary Figure S1. In this study, we examined the role of DIR1 in the priming of guard cells during SAR using the dir1-1 T-DNA insertion mutant (Maldonado et al., 2002) and its WT ecotype WS. As previously reported for the Arabidopsis Columbia ecotype (Melotto et al., 2008; David et al., 2020), the basal immune response of the mock-primed WT WS stomata closed after $1 \mathrm{~h}$ of exposure to Pst and then reopened after $3 \mathrm{~h}$. In contrast, Pst-primed WT WS leaves (distant, non-injected leaves of Pst-primed plants) did not exhibit such stomatal immune responses and maintained a small stomatal aperture during the entire period of Pst exposure, similar to that previously observed in the WT Columbia (David et al., 2020) (Figure 1A). There was no significant difference in the stomatal aperture from the Pstprimed leaves taken at 0,1 , and $3 \mathrm{~h}$ after $P s t$ exposure (Figure 1B). However, guard cells of distant leaves of dir1-1 mutant plants showed an altered response to priming and 


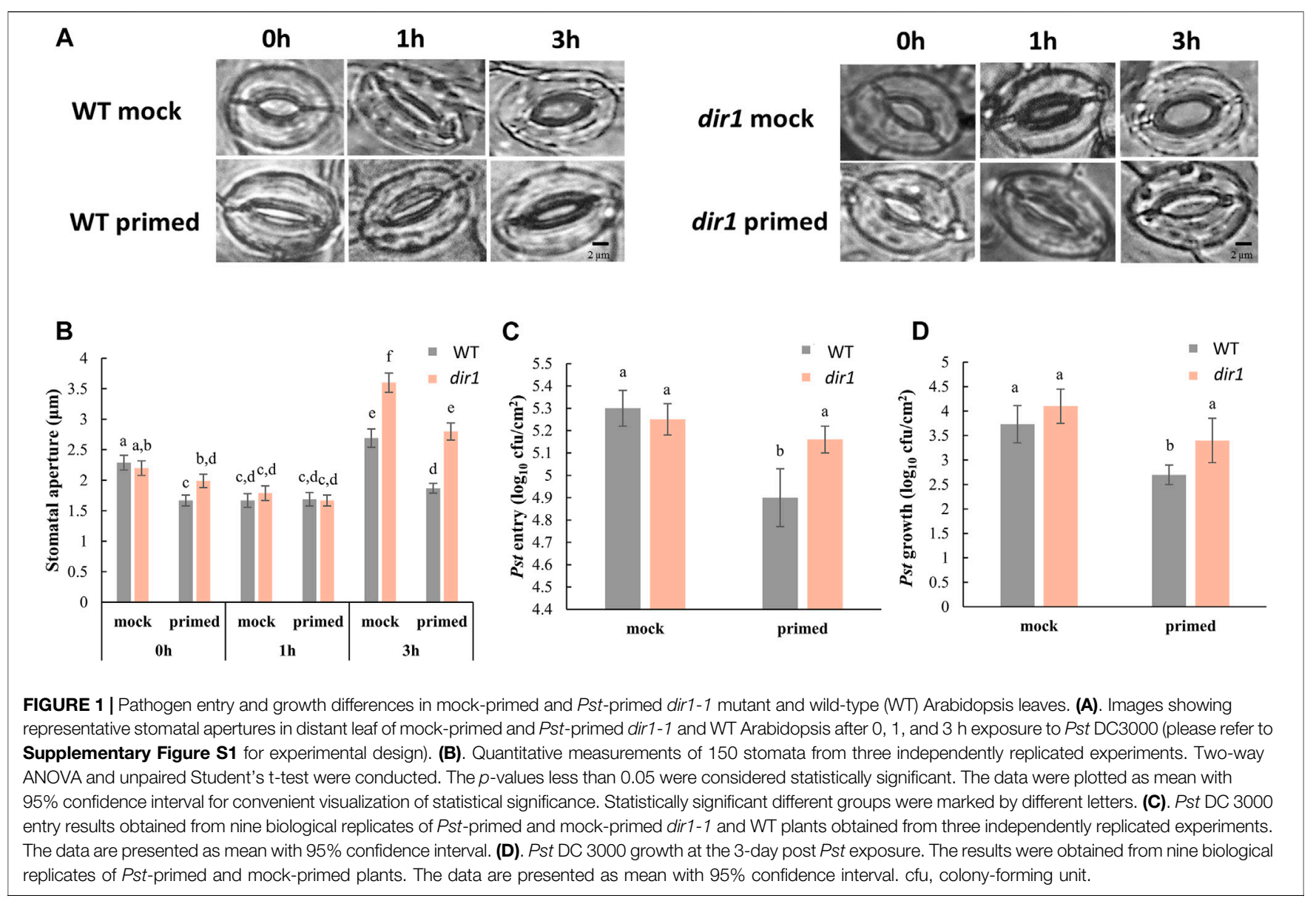

remained more open at 0 and $3 \mathrm{~h}$ compared to WT. It can be noted that due to the perception of pathogen-associated molecular patterns (PAMPs), the 1-h mock-primed and Pstprimed WT and dirl-1 apertures are similar. Specifically, the average stomatal aperture of Pst-primed dir1-1 leaves was 1.99 vs. $1.67 \mu \mathrm{m}$ in $\mathrm{WT}$ at $0 \mathrm{~h}$. At $3 \mathrm{~h}$ it was $2.80 \mathrm{vs} .1 .87 \mu \mathrm{m}$ for dir $1-1$ and $\mathrm{WT}$, respectively. Interestingly, mock-primed dir1-1 also showed a larger stomatal aperture at $3 \mathrm{~h}$ after exposure to Pst when compared to mock-primed WT with an average of 3.60 and $2.69 \mu \mathrm{m}$, respectively (Figure 1B).

In the dir1-1 mutant, we found that both the control (mockprimed) and Pst-primed dir1-1 stomatal aperture differed from WT stomata with the same Pst treatments (Figure 1). In control plants (mock-primed), we found that the initial $(0 \mathrm{~h})$ and PAMP responses $(1 \mathrm{~h})$ of the dirl-1 stomata were not statistically different from those of WT stomata to Pst exposure. However, at $3 \mathrm{~h}$ after exposure to Pst, the dir1-1 mutant displayed a wider stomatal phenotype, indicating that COR secreted from Pst had a greater effect on the dir1-1 stomata than on the WT (Figures 1A,B). The effect of priming on the stomatal aperture of dir1-1 was also different than that of WT. Intriguingly, the dir1-1 Pstprimed stomata apertures in distant leaves at $0 \mathrm{~h}$ were significantly narrower than the control (mock-primed) dir1-1 stomata, but less narrow than the WT Pst-primed stomata. The mock-primed WT and dir1-1 stomata apertures had no significant difference at $0 \mathrm{~h}$ (2.29- and 2.20- $\mu \mathrm{m}$ averages, respectively). After priming, the WT stomatal aperture decreased to $1.63 \mu \mathrm{m}$, but the dir1-1 stomata aperture was reduced to only $1.99 \mu \mathrm{m}$, making the Pst-primed dir1-1 stomata apertures significantly different from both the control (mock-primed) dir1-1 stomata and the Pst-primed WT stomata. The 1-h response to PAMPs from Pst was similar regardless of genotype (WT vs. dir1-1) or priming, showing the specific response of stomatal closure after PAMP perception. However, at $3 \mathrm{~h}$ post Pst treatment, the dirl-1 Pst-primed stomata phenotype is different from both the dir1-1 control and the WT Pst-primed stomata. Like the stomatal phenotype seen at $0 \mathrm{~h}$, the dir1-1 Pst-primed stomata had a narrower aperture $(2.8 \mu \mathrm{m})$ than the dir1-1 mock-primed stomata $(3.6 \mu \mathrm{m})$ but were less narrow than the WT Pst-primed stomata $(1.87 \mu \mathrm{m})$. This suggests that although the dir1-1 mutant appears to be less resistant to the COR from the Pst than the WT, it does have improved resistance to COR with priming (Figures 1A,B).

Pst entry and Pst growth are not significantly different in the mock-primed dir1-1 vs. WT plants, consistent with previous evidence that the dirl-1 mutant is defective in SAR response, but not in basal pathogen response (Maldonado et al., 2002). Importantly, the altered stomatal phenotype of dir1-1 is directly associated with Pst entry into the apoplastic spaces of the leaves and reduced stomatal immunity (Figure 1C). There was no 
significant difference in the number of $P s t$ that were able to enter the apoplast of mock-primed WT, mock-primed dir1-1, or Pstprimed dir1-1 leaves. Only Pst-primed WT stomata were able to reduce Pst entry after $3 \mathrm{~h}$ exposure to the bacterial pathogen (Figure 1C). Although overall immune response of the dir1-1 mutant is reduced, dir1-1 plants are still able to mount a SAR response, as indicated by the overall decreased Pst growth after 3 days of exposure in the dir1-1 Pst-primed leaves compared to the WT leaves (Figure 1D).

dir1-1 is deficient in both local and systemic guard cell immune responses. Although SAR has largely been studied at the level of leaf or whole plant level, we have recently shown evidence that SAR affects guard cell response to the bacterial pathogen Pst (David et al., 2020). DIR1 is required for movement of several chemically diverse SAR signals including DA, G3P, AzA, and possibly MeSA (Park et al., 2009; Adam et al., 2018). As we have recently reported stomatal movement and guard cell molecular changes underlying stomatal SAR responses (David et al., 2020), here we first characterized the stomatal movement phenotype of the dir1-1 mutant versus WT in response to Pst. Results from our work and previous studies (Melotto et al., 2008; Pang et al., 2020) clearly showed that stomatal guard cells from different ecotypes of Arabidopsis (WS and Columbia) exhibited similar basal immune responses. After priming for 3 days, stomata in distant leaves from the WT WS had an initial narrow aperture compared to control (mock-primed) stomata, and they maintain this narrow aperture during PAMP perceptions at $1 \mathrm{~h}$ and also at $3 \mathrm{~h}$ after $P s t$ treatment (Figure 1). This result is also similar to the WT Columbia plants (David et al., 2020).

In the dir1-1 mutant, at $3 \mathrm{~h}$ after exposure to Pst the dir1-1 mutant displayed a larger stomatal aperture, suggesting that COR secreted from Pst had a greater effect on the dir1-1 guard cells than on the WT. The effect of priming on the dir1-1 stomata was also different from that on the WT stomata. The dir1-1 Pstprimed stomata apertures at $0 \mathrm{~h}$ were narrower than the mockprimed dir1-1, but less narrow than the WT Pst-primed stomata. At $3 \mathrm{~h}$ post Pst treatment, the dir1-1 Pst-primed stomatal aperture is smaller than mock-primed, but less narrow than the WT Pstprimed. The altered stomatal aperture of dir1-1 directly associates with Pst entry into the apoplastic space (Figure 1). Clearly, although the dir1-1 mutant appears to be less resistant to the COR (from Pst) than the WT, it does have improved resistance after priming. This result is consistent with previous literature, which showed a partial SAR-competent phenotype of dir1-1 (Champigny et al., 2013). Although the partial SAR-competent phenotype of dir1-1 was able to reduce the Pst growth, it did not decrease the entry of Pst via the stomatal pores. Therefore, dir1-1 is deficient in both local and systemic guard cell immunity.

Differentially abundant proteins in the Pst-primed dir1-1 and WT guard cells are observed. Proteomic analysis of WT versus dir1-1 Pst-primed guard cell samples taken from distal leaves 3 days after Pst treatment identified 2,229 proteins, each with more than one unique peptide (1\% FDR). Of the identified proteins, 155 showed differential abundances in the Pstprimed WT guard cells compared to the dir1-1 guard cells, with 25 which increased in abundance and 130 which decreased in abundance, by $>2$-fold and a $p$-value $<0.05$ (Figure 2A). Of the differentially abundant proteins in dir1-1 Pst-primed versus (vs.) WT Pst-primed, only seven were differentially abundant in dir1-1 mock-primed vs. WT mockprimed, indicating that most changes in protein abundance were due to SAR response, rather than to genotype differences. Of the 155 differential proteins, 76 were mapped to the Arabidopsis KEGG pathway. Again, only three of the 76 were differentially abundant in dir1-1 mock-primed vs. WT mock-primed. They were phosphoribosylformylglycinamidine cyclo-ligase (mapped to purine metabolism and biosynthesis of secondary metabolites), vacuolar-sorting protein (in endocytosis pathway), and $40 \mathrm{~S}$ ribosomal protein (in the ribosome pathway). Interestingly, about $88 \%$ of the identified proteins in this study could be found in previously published guard cell transcriptomics and proteomics papers (Supplementary Table S1), highlighting that highly enriched guard cell samples were used in this study.

Based on biological functions, most differentially abundant proteins can be broadly categorized into two groups: carbon metabolism-related and amino acid biosynthesis-related. Carbon metabolism-related included 42 proteins from carbon metabolism (13), carbon fixation in photosynthetic organisms (6), glycolysis/gluconeogenesis (6), fructose and mannose metabolism (5), glyoxylate and dicarboxylate metabolism (3), pyruvate metabolism (3), starch and sucrose metabolism (3), and photosynthesis (3). Amino acid biosynthesis-related included cysteine and methionine metabolism (9), and purine metabolism (5). Notably, differentially abundant proteins also grouped into inositol phosphate metabolism 3) related to calcium signaling, terpenoid backbone biosynthesis 3) related to sterols and carotenoids, and glutathione metabolism 3) related to redox signaling (Figure 2A).

Carbon metabolism-related proteins included fructosebisphosphate aldolase 3 (FBA3), an enzyme involved in the reversible cleavage of fructose-1,6-bisphosphate into dihydroxyacetone phosphate (DHAP) and glyceraldehyde-3phosphate (GA3P), and two triosephosphate isomerases (TIM and TPI) that catalyze the reversible isomerization between DHAP and GA3P. There, three enzymes exhibited 2-fold decreases in the dir1-1 Pst-primed guard cells compared to WT. Because of the overlap of the carbon metabolism and amino acid biosynthetic KEGG pathways, some differentially abundant proteins were involved in both biological processes, including a pyruvate kinase family protein $(\mathrm{PKPa})$ and an enolase (LOS2). Both were decreased more than 2-fold in dir1-1 Pstprimed guard cells compared to WT Pst-primed (Figure 3).

The second largest group of differential proteins is related to amino acid metabolism and other pathways with 32 differential proteins between dir1-1 vs. WT Pst-primed guard cells. Some of the proteins are also identified in the KEGG biosynthesis of secondary metabolites. For example, maternal effect embryo arrest 32 (MEE32) is a putative dehydroquinate dehydratase and putative shikimate dehydrogenase. It is found in multiple KEGG pathways including biosynthesis of amino acids, metabolic pathways, phenylalanine, tyrosine, and tryptophan biosynthesis, and biosynthesis of secondary metabolites. Another example is aconitase $2(\mathrm{ACO} 2)$ which is also found in multiple KEGG 


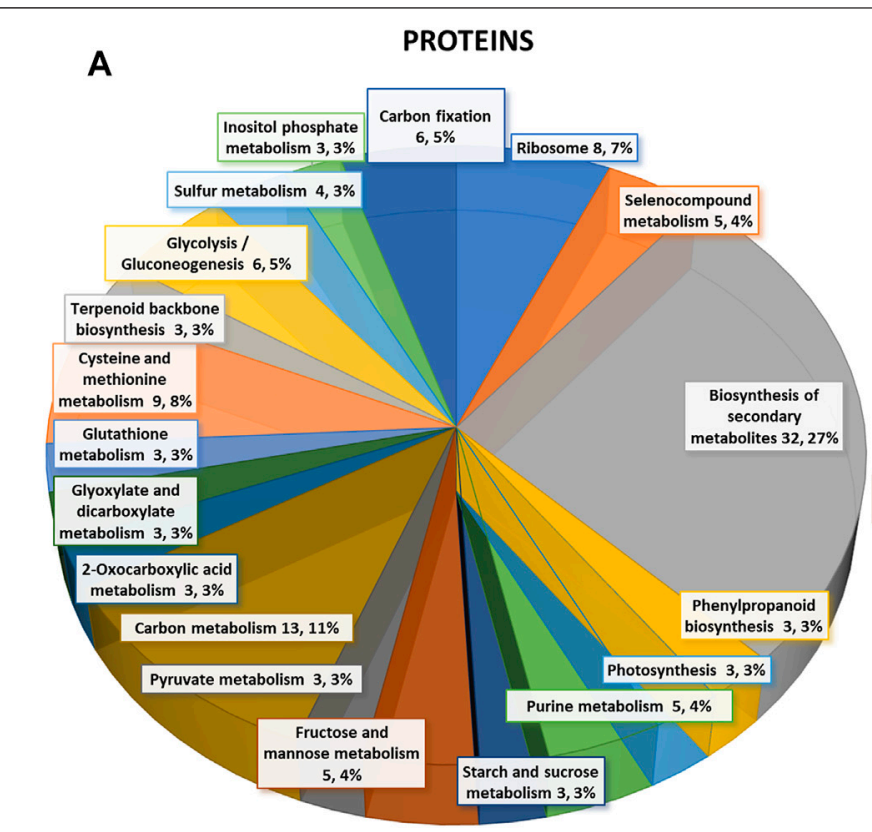

B

METABOLITES

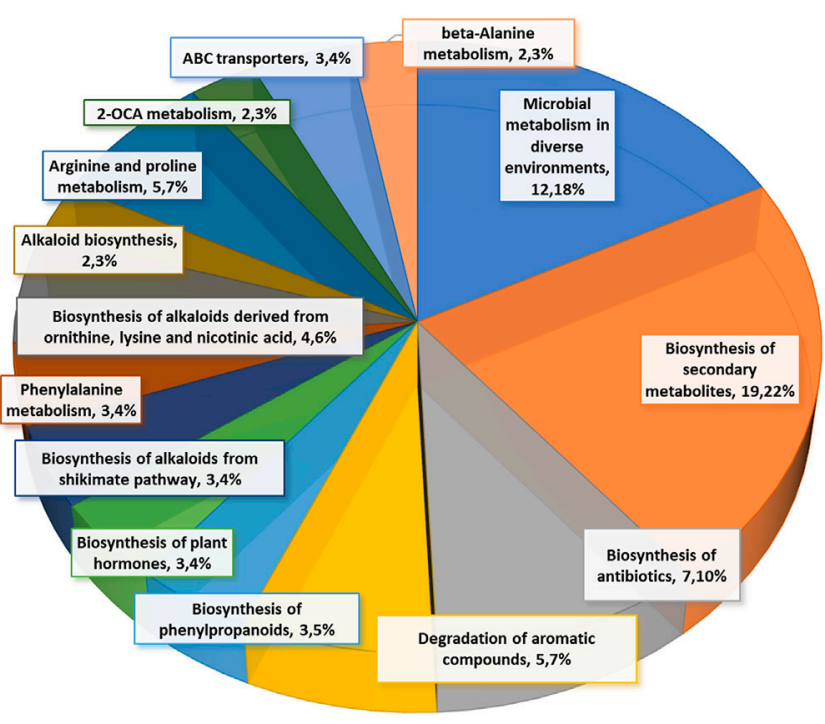

FIGURE 2 | Differential changes of proteins and metabolites in mock-primed and Pst-primed dir1-1 mutant and WT guard cells. (A). Biological function proteins found in KEGG pathways that are differentially abundant in WT versus dir1-1 Pst-primed guard cells. (B). Biological functions metabolites found in KEGG pathways that are differentially abundant in WT versus dir1-1 Pst-primed guard cells.

pathways, e.g., biosynthesis of secondary metabolites, carbon metabolism, 2-oxocarboxylic acid metabolism, glyoxylate and dicarboxylate metabolism, biosynthesis of amino acids, citrate cycle (TCA cycle), and metabolic pathways.

Amino acid biosynthesis-related proteins included aberrant growth and death 2 (AGD2), which encodes a diaminopimelate aminotransferase involved in disease resistance against Pst and the lysine biosynthesis via diaminopimelate; methionine synthase 2 (MS2), cysteine synthase C1 (CYSC1), and cystathionine betalyase $(\mathrm{CBL})$, which are all involved in cysteine and methionine biosynthesis; and an acetylornithine deacetylase involved in arginine biosynthesis. All mentioned amino acid biosynthesisrelated proteins were decreased more than 2-fold in dir1-1 Pstprimed guard cells compared to WT Pst-primed (Figure 3). Differentially abundant proteins involved in redox pathways included glutathione synthetase 2 (GSH2) and glutathione S-transferase TAU 20 (GSTU20) related to redox signaling (Mallikarjun et al., 2012). A pathway enrichment analysis was conducted for the differentially abundant proteins using AGRIGO Singular Enrichment Analysis (SEA) (Du et al., 2010) (Supplementary Figures S2 and S3). A graphical representation of GO hieratical groups with all statistically significant terms classified levels of enrichment with corresponding colors. The functional enrichment was found in three general groups including response to stimulus, amino acid metabolic processes, and carbohydrate metabolic processes (Supplementary Figure S2). AGRIGO singular enrichment analysis for cellular components revealed enrichment in intracellular organelles including intracellular membranebounded organelles, plastids, and chloroplast stroma (Supplementary Figure S3).
Differential metabolites in the Pst-primed dir1-1 and WT guard cells were observed. A total of 728 metabolites were identified, and 55 metabolites showed significant changes after the priming treatment in the dir1-1 versus WT guard cells, with 16 increased and 39 decreased in abundance, by $>2$-fold and a $p$-value $<0.05$ (Figure 2B). Of these differential metabolites, 34 were mapped to KEGG pathways. When grouping by biological function, the largest group of differentially abundant metabolites found in KEGG pathways was related to biosynthesis of secondary metabolites 19) (Figure 2B).

Several differential metabolites are involved in amino acid biosynthesis and hormone metabolism. For example, SA was decreased by more than 40-fold in the dir1-1 Pst-primed guard cells compared to WT samples (Figure 4). However, it should be noted that in dir1-1 mock-primed versus WT mock-primed, SA abundance is also decreased by more than 40 -fold. Metabolites involved in lysine biosynthesis were decreased more than 2-fold in the dir1-1 Pst-primed guard cells compared to WT. They included gly-leu, niacin, acetyl-leucine, and desaminotyrosine. Metabolites involved in arginine biosynthesis were also changed. For example, pyroglutamic acid decreased more than 2-fold, and aminolevulinic acid increased more than 4-fold in the dir1-1 Pstprimed guard cells compared to WT guard cells. Malic acid, which is related to carbon metabolism, was increased 1.8 -fold in dir1-1 versus WT Pst-primed guard cells but was decreased by nearly 2 -fold in dir1-1 vs. WT mock-primed. When malic acid in the guard cell is pumped out to the apoplast, water moves out reducing turgor pressure in the guard cells and closing the stomata (Santelia and Lawson, 2016).

Proteomic and metabolomics results indicate that DIR1 affects guard cell carbon metabolism and amino acid biosynthesis during 


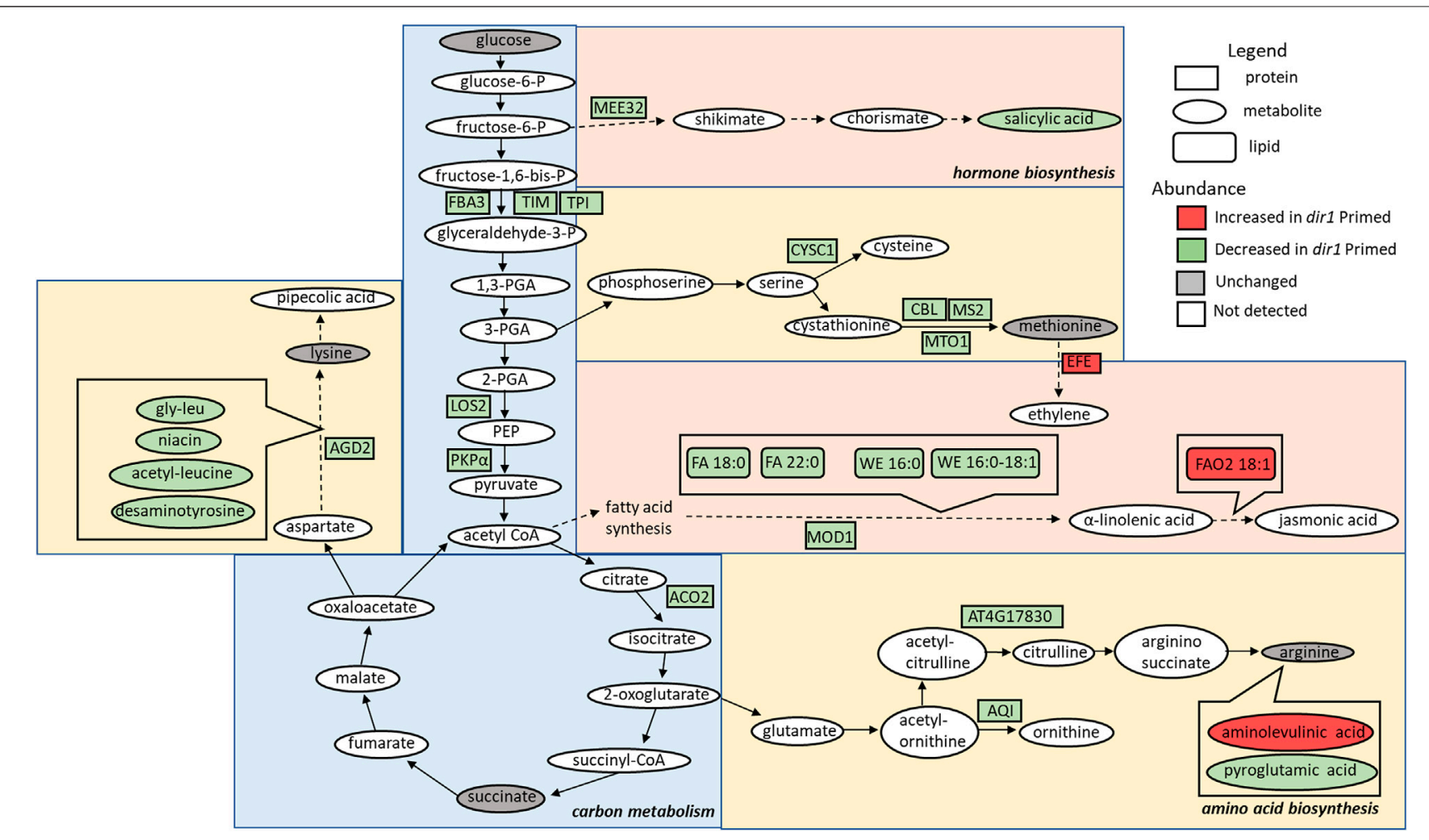

FIGURE 3 | Overview of the role of DIR1 in carbon metabolism, amino acid biosynthesis, and hormone biosynthesis in guard cells during systemic defense response. Loss of DIR1 results in altered abundance of proteins, metabolites, and lipids involved in carbon metabolism, amino acid biosynthesis, and biosynthesis of plant hormones and secondary metabolites. Proteins that were decreased in dir1-1 guard cells in the carbon metabolism metabolic pathway included FBA3, TIM, TPI, LOS2, PKPa, and ACO2. Proteins that were decreased in dir1-1 guard cells in the amino acid biosynthesis metabolic pathways included AGD2, CYSC1, CBL, MS2, MTO1, AT4G17830, and AQI, and decreased metabolites in these pathways included gly-leu, niacin, acetyl-leucine, desaminotyrosine, and pyroglutamic acid. One increased metabolite in dir1-1 guard cells in the arginine biosynthesis pathway was aminolevulinic acid. Proteins that were decreased in dir1-1 guard cells in the biosynthesis of hormones and secondary metabolites metabolic pathways included MEE32 and MOD1, and decreased metabolites and lipids in these pathways included salicylic acid, stearic acid (FA 18:0), behenic acid (FA 22:0), cetyl oleate (WE 16:0/18:1), and ethyl myristate (WE 16:0). One protein, EFE, and one lipid, FAO2 18: 1, were increased in these pathways in the dir1-1 Pst-primed guard cells versus WT Pst-primed guard cells. Please refer to Supplementary Table S3 for abbreviations.

SAR. The majority of the differential proteins and metabolites were in the carbon metabolism, amino acid biosynthesis, and secondary metabolite biosynthesis pathways (Figures 2, 3). Most of the molecules were lower in the Pst-primed dir1-1 guard cells than the Pst-primed WT guard cells. These results indicate that DIR1-dependent SAR is necessary for regulation of amino acid biosynthesis and secondary metabolites in guard cells. It also indicates that guard cells attenuate their carbon metabolic pathways to divert resources to amino acid biosynthesis in response to priming in $\mathrm{WT}$ and that this process is at least partially dependent on DIR1 in guard cells. In addition, the differential proteins enriched for plastid and chloroplast components again support alterations in carbon metabolic pathways induced by SAR. Similarly, distant leaves of $A$. thaliana after infection by $P$. syringe have shown alterations in primary metabolism, including nitrogen metabolism and amino acid content (Schwachtje et al., 2018). We propose that reorganization of primary metabolism and amino acid biosynthesis during SAR is partially dependent on DIR1. With data from both proteomics and metabolomics, the changes of at least 15 proteins were correlated with the metabolite changes in the same KEGG pathways (Supplementary Table S2). One metabolite aminolevulinic acid increased in Pst-primed dir1-1 compared to Pst-primed WT, but the proteins in the same KEGG pathway decreased. The rest of the proteins and metabolites showed the same trend of changes, suggesting translational level regulation (Supplementary Table S2).

One interesting aspect of our results is that we did not identify changes in pathogenesis-related (PR) proteins in the dir1-1 Pstprimed guard cells. Similarly, the abundance of AzA was not significantly different in the Pst-primed dir1-1 versus WT guard cells. On the other hand, the key regulatory SAR metabolite SA showed a 40 -fold decrease in the Pst-primed dir1-1 vs. WT guard cells. Previously, we reported that Pst-primed guard cells in uninoculated leaves of Arabidopsis narrowed stomatal apertures and reduced entry of Pst into the leaves and had increased SA in Pst-primed guard cells compared to mockprimed guard cells (David et al., 2020). The lower SA in the 

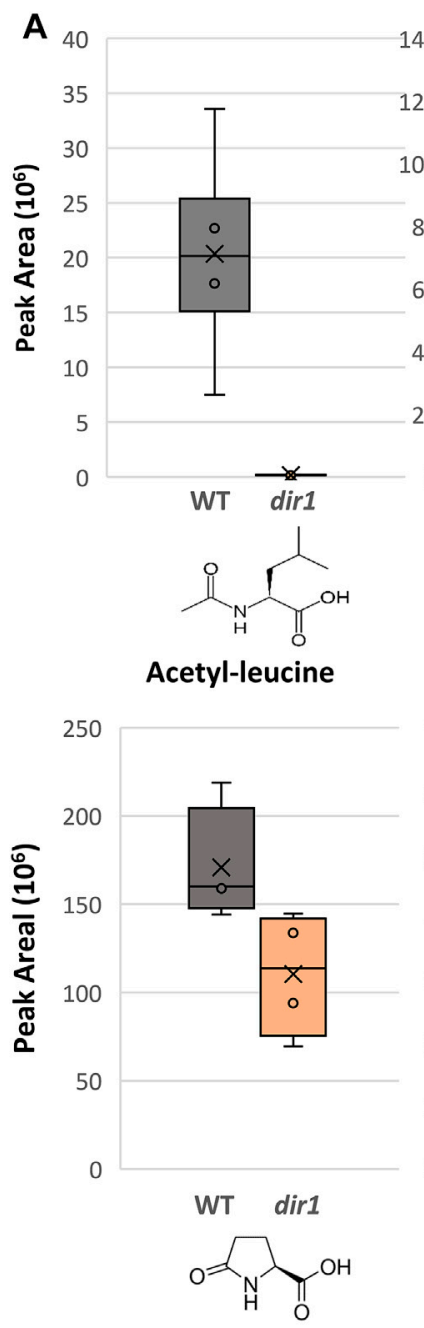

Pyroglutamic acid

B

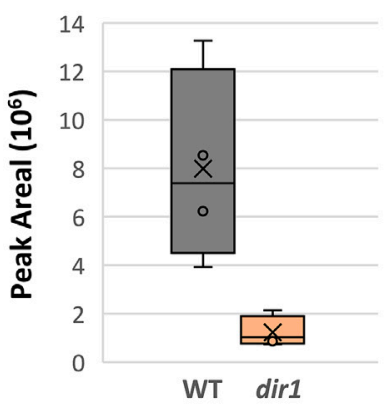

䄯

Stearic acid-FA 18:0

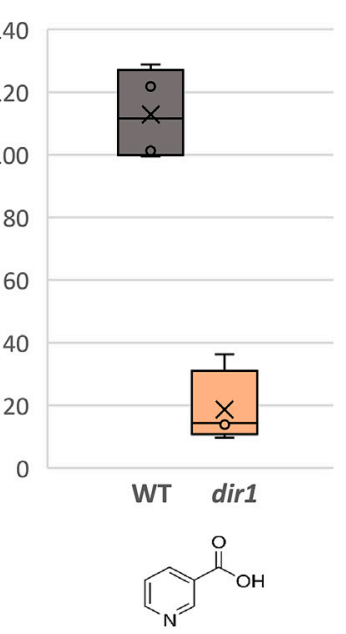

Niacin

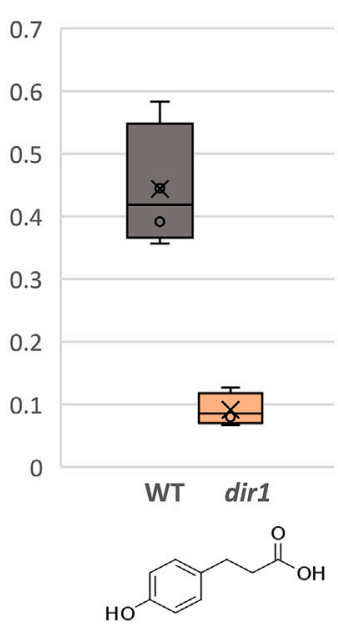

Desaminotyrosine

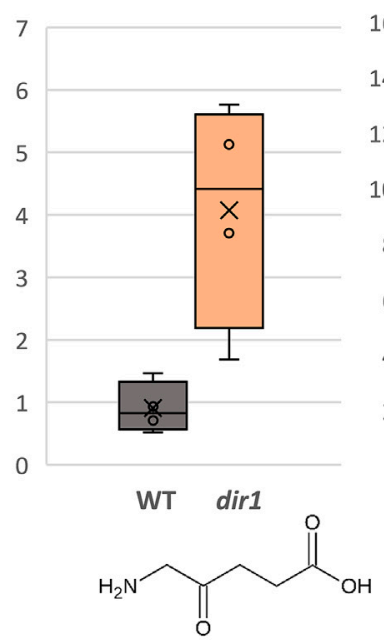

Aminolevulinic acid

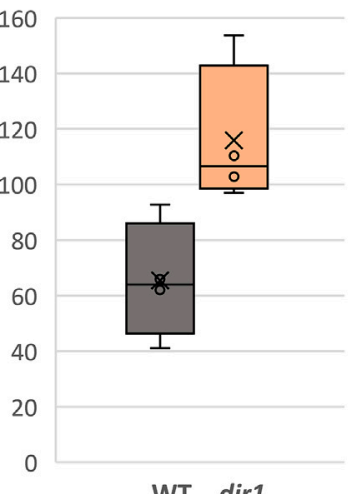

WT dir1<smiles>O=C(O)CC(O)C(=O)O</smiles>

Malic Acid

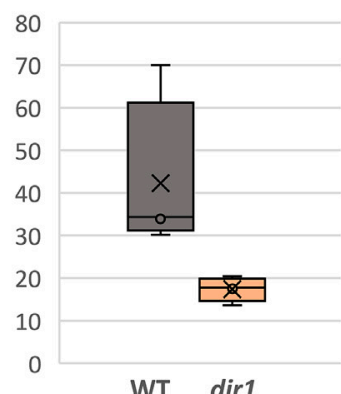

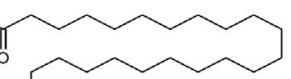

Behenic acid-FA 22:0

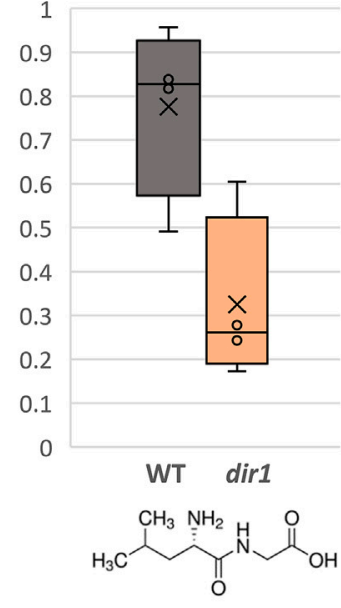

Gly-Leu

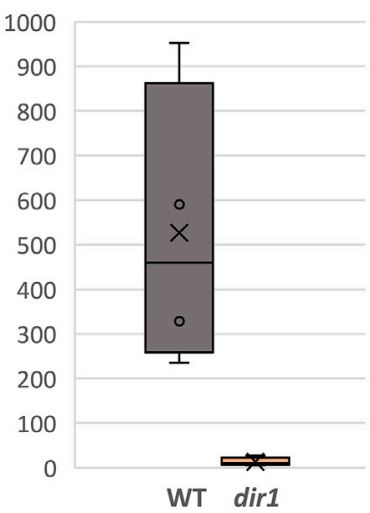<smiles>O=C(O)c1ccccc1O</smiles>

Salicylic acid
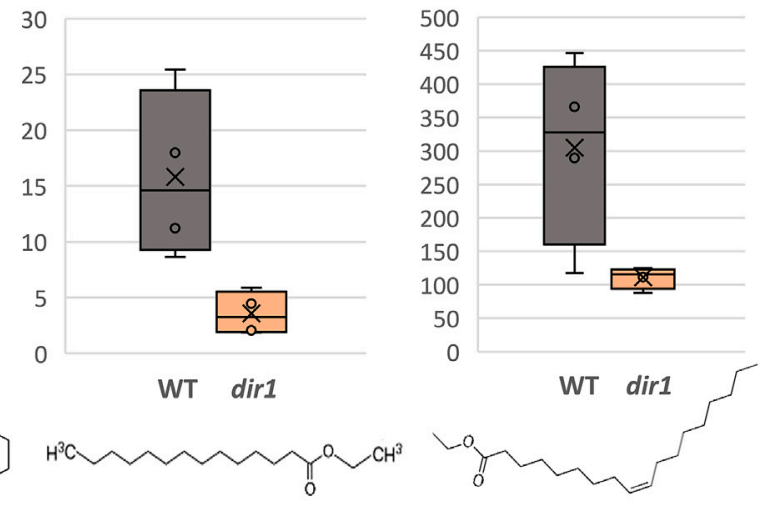

Ethyl myristate-WE 16:0 Cetyl oleate-WE 16:0/18:1

FIGURE 4 | Differentially abundant metabolites identified in dir1-1 and Pst-primed guard cells. (A). Box plots showing decreases of acetylleucine, niacin, desaminotyrosine, gly-leu, pyroglutamic acid, and salicylic acid, as well as increases aminolevulinic acid and of malic acid in dir1-1 versus WT guard cells. The error bars are standard deviation of the mean. (B). Box plots showing decreases of two long-chain fatty acids, stearic acid (FA 18:0) and behenic acid (FA 22:0), and two wax esters, cetyl oleate (WE 16:0/18:1) and ethyl myristate (WE 16:0) decreased > 2-fold in the dir 1-1 versus WT guard cells. Chemical structures of stearic acid (FA 18: 0), behenic acid (FA 22:0), cetyl oleate (WE 16:0/18:1), and ethyl myristate (WE 16:0) are shown. 
Pst-primed dir1-1 guard cells associates well with our previous findings and suggests that DIR1 is required to transmit the longdistance SAR signal to the guard cells in uninfected leaves and increase SA in the Pst-primed guard cells. Recently, translocation of SA from primary infected tissue to distal uninfected leaves was shown to likely occur via the apoplastic space between the cell wall and the plasma membrane (Lim et al., 2016). Unlike the SAR-induced signals G3P and AzA, for which evidence exits are preferentially transported via symplastic transport and through plasmodesmata, pathogen infection resulted in increased SA accumulation in the apoplastic compartment, and SARinduced accumulation was unaffected by defects in symplastic transport via plasmodesmata (Lim et al., 2016). Mature guard cells have callose depositions that block plasmodesmata (Lee and Lu, 2011). Thus, SAR signals that can be transported via the apoplast, rather than the symplast, would logically be able to affect the guard cells during SAR, much like ABA in the apoplast can also affect guard cells (Wittek et al., 2014). Alternatively, SA could be de novo synthesized in the Pst-primed guard cells. This SA biosynthesis is also affected by DIR1 mutation. How DIR1 regulates $\mathrm{SA}$ biosynthesis is not known.

Differential lipids in the Pst-primed dir1-1 and WT guard cells were observed. A total of 1,197 lipids were identified, and 88 lipids showed significant changes in guard cells after the priming of the dir 1-1 vs. WT guard cells (with 37 increased and 49 decreased by $>2$ fold). Of the differential lipids, 15 were mapped to KEGG pathways and their biological functions largely fell into two categories: biosynthesis of fatty acids and biosynthesis of secondary metabolites. Notably these lipids included FAO2 18:1, isoleukotoxin diol (DiHOME) involved in linoleic acid metabolism (a precursor for jasmonic acid). It was increased 2.1fold in the dir1-1 vs. WT Pst-primed guard cells. We also found two long-chain fatty acids (FA) including stearic acid (FA 18:0) and behenic acid (FA 22:0) and two wax esters (WE) including cetyl oleate (WE 16:0/18:1) and ethyl myristate (WE 16:0). They were all decreased more than 2-fold in the Pst-primed dir1-1 vs. WT guard cells (Figures 3, 4). Ethyl myristate is a long-chain fatty acid ethyl ester resulting from the condensation of the carboxy group of myristic acid with the hydroxy group of ethanol. Palmityl oleate is a wax ester obtained by the condensation of hexadecan-1-ol with oleic acid. Interestingly, both stearic acid and behenic acid were not significantly changed in the dir1-1 mock-primed vs. WT mock, indicating that this change in FA amount is due to priming, further supporting that they may be the long-chain lipid signals potentially transported by DIR1. As to the two wax esters (cetyl oleate and ethyl myristate), they were already more than 2-fold reduced in dir1-1 mock-primed vs. WT mock-primed, indicating genotypic difference rather than priming effect.

Previously, we found that fatty acids were increased in the Pst-primed WT guard cells (David et al., 2020). Here we compared the levels of lipids found in Pst-primed WT guard cells to those in the dir1-1 mutant. Our goal was to identify lipids in guard cells that are dependent on DIR1 during priming. DIR1 has been characterized as an LTP, and the core of its structure forms a left-handed super helical arrangement of four $\alpha$-helices building the hydrophobic central cavity. Lascombe et al. (2008) demonstrated that DIR1 showed a greater affinity for LPCs with fatty acid chain lengths with $>14$ carbon atoms and that nonpolar C18 fatty acid tails were completely buried within the barrel structure of the DIR1 protein, presumably allowing non-polar fatty acids to be transported in polar cellular environments. Here, lipidomic results revealed four longchain fatty acids associated with DIR1. The two long-chain fatty acids (stearic acid (FA 18:0) and behenic acid (FA 22:0)) and two wax esters (cetyl oleate (WE 16:0/18:1) and ethyl myristate (WE 16:0) ) were all decreased $>2$-fold in the dir1-1 guard cells compared to WT guard cells (Figures 3, 4). As both stearic acid and behenic acid were not significantly changed in dir1-1 mock-primed vs. WT mock-primed, this change in FA levels is likely due to priming, further supporting that they may be the long-chain lipid signals transported by DIR1. Further analysis is required to determine the relationship between DIR1 and these long-chain fatty acids. It is reasonable to propose that DIR1 may transfer stearic and behenic acid to guard cells during SAR. Previously, we identified an increase in palmitic acid and its derivative 9-(palmitoyloxy) octadecanoic acid in Pst-primed WT guard cells and proposed that fatty acids could allow for the development of lipid rafts or other alterations of membrane structure in guard cells, modulating stomatal immune responses (David et al., 2020).

Plant wax esters are neutral lipids with long-chain (C16 and C18) or very-long-chain (C20 and longer) carbon structures and are mostly found in cuticles where they provide a hydrophobic coating to shoot surfaces ( $\mathrm{Li}$ et al., 2008). Recently, the cuticle has been indicated to regulate transport of SA from pathogeninfected to uninfected parts of the plant via the apoplast during SAR (Lim et al., 2020). Lim et al. (2020) found that cuticle-defective mutants with increased transpiration and larger stomatal apertures reduced the apoplastic transport of SA and caused defective SAR response. It is interesting to note that our results demonstrate that WT stomata maintain narrow stomata apertures after priming, potentially to reduce transpiration and increase water potential, and possibly routing SA to the apoplast. The dirl-1 mutant, on the other hand, had larger stomatal apertures, perhaps resulting in defect in SA movement in the apoplast. It is not known whether the mutant has defect in cuticle structure due to the decreases of wax esters (cetyl oleate and ethyl myristate). However, since the decreased cetyl oleate and ethyl myristate in dir1-1 guard cells after priming were already $>2$-fold reduced in dir1-1 mockprimed vs. WT mock-primed, this was a genotypic difference, rather than a result of priming. If, as reported by Lim et al. (2020), defects in the cuticle reduce transport of SA, the reduced wax esters in dir1-1 vs. WT could explain the reduce SA in dir1-1 guard cells (both mock-primed and Pst-primed) and contribute to the SAR defect of the dir1-1 mutant.

One cuticle-defective mutant was a knockout of MOD1, an enoyl-[acyl-carrier-protein] reductase which transports a growing FA chain between enzyme domains of FA synthase during FA biosynthesis (Nguyen et al., 2014; He and Ding, 2020). The $\bmod 1$ mutant is defective in the key FA biosynthetic enzyme enoyl-ACP reductase and has reduced levels of multiple FA species and total lipids (Lim et al., 2020). Interestingly, we also found that MOD1 was $>2$-fold lower in 
A

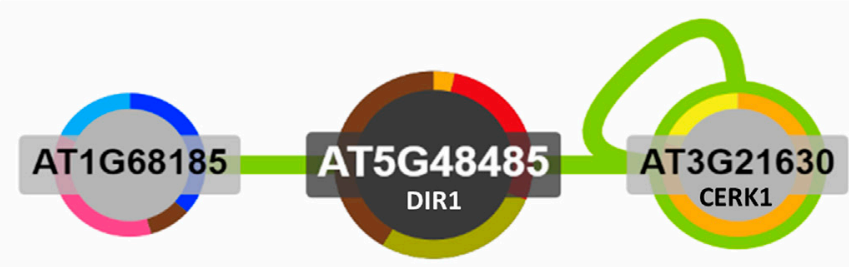

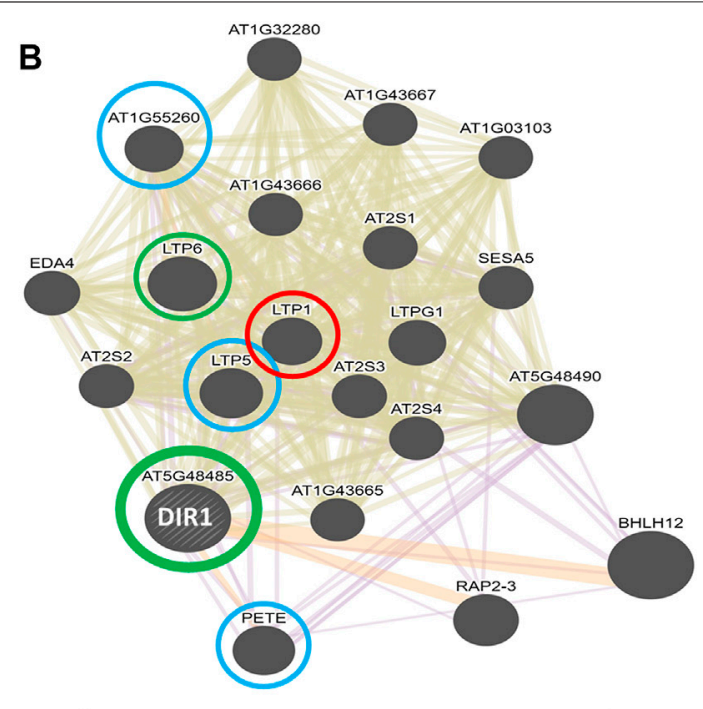

NETWORKS:

Genetic interactions

Co-expression

Predicted

FIGURE 5 | Identification of potential interacting proteins with DIR1. (A). Protein interaction image was generated using Interaction Viewer at bar.utoronto.ca/ eplant. Border color indicates protein location. Green lines indicate protein and DNA interactions that have been experimentally determined. (B). GeneMANIA tool from bar.utoronto.ca/eplant was used to predict other genes/gene products associated with DIR1 (AT5G48485). Predicted, co-expression, and genetic interaction networks found associated genes/gene products. Proteins identified in guard cell samples are circled. Circle colors indicate increased (red), decreased (green), or unchanged (blue) proteins in dir1-1 versus WT Pst-primed guard cells.

dir1-1 guard cells versus WT guard cells after priming (Figure 3). This result supports our previous results that FA synthesis plays a key role in SAR priming in guard cells (David et al., 2020). However, how DIR1 affects MOD1 and FA biosynthesis awaits further investigation.

Potential DIR1-interacting proteins are shown. Using the Interaction Viewer at the Bio-Analytic Resource for Plant Biology (BAR) (bar.utoronto.ca/eplant), localizations of DIR1 and proteins that interact with DIR1 (AT5G48485) were determined (Figure 5A). Cellular localizations of DIR1 included peroxisomes, Golgi apparatus, endoplasmic reticulum, and plasma membrane. Protein-protein interactions that have been experimentally determined, indicated by the straight, green lines, occur between DIR1 and both ubiquitin-like protein (AT1G68185) and chitin elicitor receptor kinase 1 (CERK1, AT3G21630). Based on Araport 11 annotation, CERK1 is a LysM receptor-like kinase and has a typical $\mathrm{RD}$ signaling domain in its catalytic loop and possesses autophosphorylation activity. GO biological functions of CERK1 include perception and transduction of the chitin oligosaccharide elicitor in innate immune response to fungal pathogens. CERK1 is located in the plasma membrane and cytoplasm and phosphorylates LIK1, an LLR-RLK that is involved in innate immunity (Junková et al., 2021; Rebaque et al., 2021). However, neither the ubiquitin-like protein nor CERK1 was identified in our proteomics results (Supplementary Table S3).
The GeneMANIA tool was used to predict other gene products associated with DIR1. Predicted, co-expression, and genetic interaction networks found associated gene products (Figure 5B). In addition to DIR1, our proteomics identified several LTPs including LTP1, LTP5, LTP6, plastocyanin (PETE1), and LTPG6 (AT1G55260) from guard cell samples. LTPG6 is a glycosylphosphatidylinositol-anchored LTP involved in defense response to fungus. LTP1 (AT2G38540) is a non-specific LTP that binds calmodulin in a $\mathrm{Ca}^{2+}$-independent manner. LTP1 is specifically expressed in the L1 epidermal layer and is localized to the cell wall (Fahlberg et al., 2019). LTP1 ${ }^{\mathrm{RNAi}}$ lines are specifically defective in systemic, but not local, resistance to Pst, providing evidence that LTP1 may also play a role in SAR (Carella et al., 2017). LTP1, LTP5 (AT3G51600), and LTP6 (AT3G08770) are predicted to encode pathogenesis-related (PR) proteins and are members of the PR-14 protein family (Sels et al., 2008). The mRNA of LTP1 is cell-to-cell mobile (Bogdanov et al., 2016). PETE1 is one of two Arabidopsis plastocyanins (PETE1 and PETE2). Its mRNA expression is one-tenth of the level of PETE2. Although PETE2 is involved in copper homeostasis, PETE1 is not responsive to increased copper levels, but it may participate in electron transport during copper-limiting conditions (Weigel et al., 2003; Abdel-Ghany, 2009). DIR1 was not present in our dir1-1 knockout mutant samples, and LTP6 was significantly decreased in the dir1-1 versus WT after priming. LTP1 was increased in dir1-1 versus WT, 
and LTP5, PETE, and LTPG6 were unchanged during priming in dir1-1 versus WT guard cells (Figure 5B). DIR1 was associated with LTP1, LTP5, LTP6, and LTPG6 via genetic interaction networks, and with PETE1 via predicted and co-expression networks (Figure 5B).

\section{CONCLUSION}

Guard cells that control stomatal aperture respond to various abiotic and biotic signals and have membrane-bound pattern recognition receptors that perceive bacterial pathogens. One neglected area of SAR research has been the role that stomatal guard cells play in SAR. This work investigates the role of SARrelated LTP DIR1 in guard cell-specific SAR. After priming and also after exposure to the bacterial pathogen Pst, the stomata of WT remain at a narrow aperture. In contrast, the dir1-1 mutant showed defects in stomatal closure. Based on the multi-omics data, proteins and metabolites related to amino acid biosynthesis, secondary metabolism, and response to stimulus were altered in guard cells of dir1-1 compared to WT. For example, several proteins in the methionine biosynthesis pathway and a protein related to ethylene biosynthesis were decreased in the dir1-1 Pst-primed guard cells compared to WT. It is known that ethylene is biosynthesized via methionine and ethylene plays a role in SA-regulated stomatal closure by mediating ROS and nitric oxide (Wang et al., 2020). A putative shikimate dehydrogenase was also decreased in the dir1-1 guard cells after priming. As SA is a product of the shikimate pathway and was also lower in dir1-1 guard cells, the slowdown in this pathway could explain the defect of stomatal closure and defense observed in the dir1-1 mutant during priming. Our lipidomics results highlight a role for fatty acid signaling and cuticle wax esters in the Pst-primed guard cells, i.e., two long-chain (18C and 22C) fatty acids as putative lipid mobile signals and two 16C wax esters dependent on DIR1. These results are also associated with a decrease in the MOD1 in the dir1-1 guard cells. As mod1 mutants have been shown to have cuticle defects and reduced transport of SA to distal tissue during SAR, this relates to the decreased SA in the dirl-1 guard cells. Multi-omics has shown utility in discovering DIR1dependent molecular networks in stomatal immunity. The improved knowledge may facilitate effort in biotechnology and marker-based breeding for enhanced plant disease resistance.

\section{REFERENCES}

Abdel-Ghany, S. E. (2009). Contribution of Plastocyanin Isoforms to Photosynthesis and Copper Homeostasis in Arabidopsis thaliana Grown at Different Copper Regimes. Planta 229, 767-779. doi:10.1007/s00425-0080869-z

Ádám, A., Nagy, Z., Kátay, G., Mergenthaler, E., and Viczián, O. (2018). Signals of Systemic Immunity in Plants: Progress and Open Questions. Ijms 19, 1146-1166. doi:10.3390/ijms19041146

Bogdanov, I. V., Shenkarev, Z. O., Finkina, E. I., Melnikova, D. N., Rumynskiy, E. I., Arseniev, A. S., et al. (2016). A Novel Lipid Transfer Protein from the Pea Pisum

\section{DATA AVAILABILITY STATEMENT}

The datasets presented in this study can be found in online repositories. The names of the repository/repositories and accession number(s) can be found in the following: all protein MS raw data and search results have been deposited to the ProteomeXchange Consortium via the PRIDE partner repository with the data set identifier PXD024991. All metabolite and lipid MS raw data and search results have been deposited to the MetaboLights data repository with the data set identifier MTBLS2614.

\section{AUTHOR CONTRIBUTIONS}

SC and LD conceived and designed the research; LD, J.K, J.N, and CD carried out all experimental work; LD conducted the data analysis and LD and SC prepared the manuscript; and SC finalized the manuscript with input from all the co-authors.

\section{FUNDING}

This material is based upon work supported by the National Science Foundation under Grant No. 1920420. This work is also supported by United States Department of Agriculture grant no. 2020-67013-32700/project accession no. 1024092 from the USDA National Institute of Food and Agriculture.

\section{ACKNOWLEDGMENTS}

We thank Dr. Tongjun Gu from Bioinformatics Core of Interdisciplinary Center for Biotechnology Research, University of Florida, for advice on statistical analysis, and Ms. Angelica Ortega and Mr. Ivan Grela for their help with growth and maintenance of Arabidopsis plants.

\section{SUPPLEMENTARY MATERIAL}

The Supplementary Material for this article can be found online at: https://www.frontiersin.org/articles/10.3389/fmolb.2021.746523/ full\#supplementary-material

Sativum: Isolation, Recombinant Expression, Solution Structure, Antifungal Activity, Lipid Binding, and Allergenic Properties. BMC Plant Biol. 16, 107. doi:10.1186/s12870-016-0792-6

Champigny, M. J., Isaacs, M., Carella, P., Faubert, J., Fobert, P. R., and Cameron, R. K. (2013). Long Distance Movement of DIR1 and Investigation of the Role of DIR1-like during Systemic Acquired Resistance in Arabidopsis. Front. Plant Sci. 4, 230. doi:10.3389/fpls.2013.00230

Champigny, M. J., Shearer, H., Mohammad, A., Haines, K., Neumann, M., Thilmony, R., et al. (2011). Localization of DIR1 at the Tissue, Cellular and Subcellular Levels during Systemic Acquired Resistance in Arabidopsisusing DIR1:GUS and DIR1:EGFP Reporters. BMC Plant Biol. 11, 125. doi:10.1186/ 1471-2229-11-125 
Champigny, M. J., Shearer, H., Mohammad, A., Haines, K., Neumann, M., Thilmony, R., et al. (2011). Localization of DIR1 at the Tissue, Cellular and Subcellular Levels during Systemic Acquired Resistance in Arabidopsisusing DIR1:GUS and DIR1:EGFP Reporters. BMC Plant Biol. 11, 125. doi:10.1186/ 1471-2229-11-125

Chanda, B., Xia, Y., Mandal, M. K., Yu, K., Sekine, K. T., Gao, Q.-m., et al. (2011). Glycerol-3-phosphate Is a Critical mobile Inducer of Systemic Immunity in Plants. Nat. Genet. 43, 421-427. doi:10.1038/ng.798

Chaturvedi, R., Venables, B., Petros, R. A., Nalam, V., Li, M., Wang, X., et al. (2012). An Abietane Diterpenoid Is a Potent Activator of Systemic Acquired Resistance. Plant J. 71, 161-172. doi:10.1111/j.1365313x.2012.04981.x

Chester, K. S. (1933). The Problem of Acquired Physiological Immunity in Plants. Q. Rev. Biol. 8, 129-154. doi:10.1086/394430

Conrath, U. (2011). Molecular Aspects of Defence Priming. Trends Plant Sci. 16 (10), 524-531. doi:10.1016/j.tplants.2011.06.004

Conrath, U. (2006). Systemic Acquired Resistance. Plant Signaling Behav. 1 (4), 179-184. doi:10.4161/psb.1.4.3221

Cornish, K., and Zeevaart, J. A. D. (1985). Movement of Abscisic Acid into the Apoplast in Response to Water Stress in Xanthium strumarium L. Plant Physiol. 78, 623-626. doi:10.1104/pp.78.3.623

David, L., Harmon, A. C., and Chen, S. (2019). Plant Immune Responses - from Guard Cells and Local Responses to Systemic Defense against Bacterial Pathogens. Plant Signaling Behav. 14, e1588667. doi:10.1080/ 15592324.2019.1588667

David, L., Kang, J., and Chen, S. (2021). Untargeted Metabolomics of Arabidopsis Stomatal Immunity. Methods Mol. Biol. 2200, 413-424. doi:10.1007/978-10716-0880-7_20

David, L., Kang, J., Dufresne, D., Zhu, D., and Chen, S. (2020). Multi-omics Revealed Molecular Mechanisms Underlying Guard Cell Systemic Acquired Resistance. Ijms 22, 191-212. doi:10.3390/ijms22010191

Du, Z., Zhou, X., Ling, Y., Zhang, Z., and Su, Z. (2010). agriGO: a GO Analysis Toolkit for the Agricultural Community. Nucleic Acids Res. 38, W64-W70. doi:10.1093/nar/gkq310

Fahlberg, P., Buhot, N., Johansson, O. N., and Andersson, M. X. (2019). Involvement of Lipid Transfer Proteins in Resistance against a Non-host Powdery Mildew inArabidopsis Thaliana. Mol. Plant Pathol. 20, 69-77. doi: $10.1111 / \mathrm{mpp} .12740$

He, M., and Ding, N.-Z. (2020). Plant Unsaturated Fatty Acids: Multiple Roles in Stress Response. Front. Plant Sci. 11, 562785. doi:10.3389/ fpls.2020.562785

Hirano, S. S., and Upper, C. D. (2000). Bacteria in the Leaf Ecosystem with Emphasis on Pseudomonas syringae -a Pathogen, Ice Nucleus, and Epiphyte. Microbiol. Mol. Biol. Rev. 64, 624-653. doi:10.1128/MMBR.64.3.624-653.2000

Jung, H. W., Tschaplinski, T. J., Wang, L., Glazebrook, J., and Greenberg, J. T. (2009). Priming in Systemic Plant Immunity. Science 324, 89-91. doi:10.1126/ science. 1170025

Junková, P., Neubergerová, M., Kalachova, T., Valentová, O., and Janda, M. (2021). Regulation of the Microsomal Proteome by Salicylic Acid and Deficiency of Phosphatidylinositol-4-kinases $\beta 1$ and $\beta 2$ in Arabidopsis thaliana. Proteomics 21, 2000223. doi:10.1002/pmic.202000223

Kang, J., David, L., Li, Y., Cang, J., and Chen, S. (2021). Three-in-one Simultaneous Extraction of Proteins, Metabolites and Lipids for Multi-Omics. Front. Genet. 12, 635971. doi:10.3389/fgene.2021.635971

Lascombe, M.-B., Bakan, B., Buhot, N., Marion, D., Blein, J.-P., Larue, V., et al. (2008). The Structure of "defective in Induced Resistance" Protein ofArabidopsis Thaliana, DIR1, Reveals a New Type of Lipid Transfer Protein. Protein Sci. 17, 1522-1530. doi:10.1110/ps.035972.108

Lascombe, M.-B., Buhot, N., Bakan, B., Marion, D., Blein, J. P., Lamb, C. J., et al. (2006). Crystallization of DIR1, a LTP2-like Resistance Signalling Protein fromArabidopsis Thaliana. Acta Cryst. Sect F 62, 702-704. doi:10.1107/ S1744309106023748

Lee, J.-Y., and Lu, H. (2011). Plasmodesmata: the Battleground against Intruders. Trends Plant Science 16 (4), 201-210. doi:10.1016/j.tplants.2011.01.004

Li, F., Wu, X., Lam, P., Bird, D., Zheng, H., Samuels, L., et al. (2008). Identification of the Wax Ester Synthase/Acyl-Coenzyme A:Diacylglycerol Acyltransferase WSD1 Required for Stem Wax Ester Biosynthesis in Arabidopsis. Plant Physiol. 148, 97-107. doi:10.1104/pp.108.123471
Lim, G.-H., Liu, H., Yu, K., Liu, R., Shine, M. B., Fernandez, J., et al. (2020). The Plant Cuticle Regulates Apoplastic Transport of Salicylic Acid during Systemic Acquired Resistance. Sci. Adv. 6, eaaz0478. doi:10.1126/sciadv.aaz0478

Lim, G.-H., Shine, M. B., de Lorenzo, L., Yu, K., Cui, W., Navarre, D., et al. (2016). Plasmodesmata Localizing Proteins Regulate Transport and Signaling during Systemic Acquired Immunity in Plants. Cell Host \& Microbe 19, 541-549. doi:10.1016/j.chom.2016.03.006

Maldonado, A. M., Doerner, P., Dixon, R. A., Lamb, C. J., and Cameron, R. K. (2002). A Putative Lipid Transfer Protein Involved in Systemic Resistance Signalling in Arabidopsis. Nature 419, 399-403. doi:10.1038/nature00962

Mallikarjun, V., Clarke, D. J., and Campbell, C. J. (2012). Cellular Redox Potential and the Biomolecular Electrochemical Series: a Systems Hypothesis. Free Radic. Biol. Med. 53, 280-288. doi:10.1016/j.freeradbiomed.2012.04.034

Martinière, A., Gibrat, R., Sentenac, H., Dumont, X., Gaillard, I., and Paris, N. (2018). Uncovering $\mathrm{pH}$ at Both Sides of the Root Plasma Membrane Interface Using Noninvasive Imaging. Proc. Natl. Acad. Sci. USA 115, 6488-6493. doi:10.1073/pnas.1721769115

Melotto, M., Underwood, W., and He, S. Y. (2008). Role of Stomata in Plant Innate Immunity and Foliar Bacterial Diseases. Annu. Rev. Phytopathol. 46, 101-122. doi:10.1146/annurev.phyto.121107.104959

Misra, B. B., and Chaturvedi, R. (2015). When Plants Brace for the Emerging Pathogens. Physiol. Mol. Plant Pathol. 92, 181-185. doi:10.1016/ j.pmpp.2015.03.004

Nandi, A., Welti, R., and Shah, J. (2004). The Arabidopsis thaliana Dihydroxyacetone Phosphate Reductase Gene SUPPRESSOR of FATTY ACID DESATURASE DEFICIENCY1 Is Required for Glycerolipid Metabolism and for the Activation of Systemic Acquired Resistance[W]. Plant Cell 16, 465-477. doi:10.1105/tpc.016907

Nguyen, C., Haushalter, R. W., Lee, D. J., Markwick, P. R. L., Bruegger, J., CaldaraFestin, G., et al. (2014). Trapping the Dynamic Acyl Carrier Protein in Fatty Acid Biosynthesis. Nature 505, 427-431. doi:10.1038/nature12810

Pang, Q., Zhang, T., Zhang, A., Lin, C., Kong, W., and Chen, S. (2020). Proteomics and Phosphoproteomics Revealed Molecular Networks of Stomatal Immune Responses. Planta 252, 66. doi:10.1007/s00425-020-03474-3

Park, S.-W., Liu, P.-P., Forouhar, F., Vlot, A. C., Tong, L., Tietjen, K., et al. (2009). Use of a Synthetic Salicylic Acid Analog to Investigate the Roles of Methyl Salicylate and its Esterases in Plant Disease Resistance. J. Biol. Chem. 284, 7307-7317. doi:10.1074/jbc.m807968200

Rebaque, D., Hierro, I., López, G., Bacete, L., Vilaplana, F., Dallabernardina, P., et al. (2021). Cell wall-derived Mixed-linked $\beta$-1,3/1,4-glucans Trigger Immune Responses and Disease Resistance in Plants. Plant J. 106, 601-615. doi:10.1111/ tpj. 15185

Ross, A. F. (1966). "Systemic Effects of Local Lesion Formation," in Viruses of Plants. Editors A. B. R. Beemster and J. Dijkstra (Amsterdam: North-Holland Publishing Company), 127-150.

Saint-Vincent, P. M., Ridout, M., Engle, N. L., Lawrence, T. J., Yeary, M. L., Tschaplinski, T. J., et al. (2020). Isolation, Characterization, and Pathogenicity of Two Pseudomonas syringae Pathovars from Populus trichocarpa Seeds. Microorganisms 8, 1137-1156. doi:10.3390/microorganisms8081137

Santelia, D., and Lawson, T. (2016). Rethinking Guard Cell Metabolism. Plant Physiol. 172, 1371-1392. doi:10.1104/pp.16.00767

Schwachtje, J., Fischer, A., Erban, A., and Kopka, J. (2018). Primed Primary Metabolism in Systemic Leaves: a Functional Systems Analysis. Sci. Rep. 8, 216. doi:10.1038/s41598-017-18397-5

Sels, J., Mathys, J., De Coninck, B. M. A., Cammue, B. P. A., and De Bolle, M. F. C. (2008). Plant Pathogenesis-Related (PR) Proteins: a Focus on PR Peptides. Plant Physiol. Biochem. 46, 941-950. doi:10.1016/j.plaphy.2008.06.011

Shah, J., and Zeier, J. (2013). Long-distance Communication and Signal Amplification in Systemic Acquired Resistance. Front. Plant Sci. 4, 1-16. doi:10.3389/fpls.2013.00030

Su, J., Zhang, M., Zhang, L., Sun, T., Liu, Y., Lukowitz, W., et al. (2017). Regulation of Stomatal Immunity by Interdependent Functions of a Pathogen-Responsive MPK3/MPK6 cascade and Abscisic Acid. Plant Cell 29, 526-542. doi:10.1105/ tpc. 16.00577

Wang, H.-Q., Sun, L.-P., Wang, L.-X., Fang, X.-W., Li, Z.-Q., Zhang, F.-F., et al. (2020). Ethylene Mediates Salicylic-Acid-Induced Stomatal Closure by Controlling Reactive Oxygen Species and Nitric Oxide Production in Arabidopsis. Plant Sci. 294, 110464. doi:10.1016/j.plantsci.2020.110464 
Weigel, M., Varotto, C., Pesaresi, P., Finazzi, G., Rappaport, F., Salamini, F., et al. (2003). Plastocyanin Is Indispensable for Photosynthetic Electron Flow in Arabidopsis thaliana. J. Biol. Chem. 278, 31286-31289. doi:10.1074/ jbc.M302876200

Wittek, F., Hoffmann, T., Kanawati, B., Bichlmeier, M., Knappe, C., Wenig, M., et al. (2014). Arabidopsis ENHANCED DISEASE SUSCEPTIBILITY1 Promotes Systemic Acquired Resistance via Azelaic Acid and its Precursor 9-oxo Nonanoic Acid. J. Exp. Bot. 65, 5919-5931. doi: $10.1093 / j x b /$ eru331

Wong, L. H., Gatta, A. T., and Levine, T. P. (2019). Lipid Transfer Proteins: the Lipid Commute via Shuttles, Bridges and Tubes. Nat. Rev. Mol. Cell Biol 20, 85-101. doi:10.1038/s41580-018-0071-5

Yu, K., Soares, J. M., Mandal, M. K., Wang, C., Chanda, B., Gifford, A. N., et al. (2013). A Feedback Regulatory Loop between G3P and Lipid Transfer Proteins DIR1 and AZI1 Mediates Azelaic-Acid-Induced Systemic Immunity. Cell Rep. 3, 1266-1278. doi:10.1016/j.celrep.2013.03.030

Zhu, M., Dai, S., and Chen, S. (2012). The Stomata Frontline of Plant Interaction with the Environment-Perspectives from Hormone Regulation. Front. Biol. 7, 96-112. doi:10.1007/s11515-012-1193-3

Zhu, M., Jeon, B. W., Geng, S., Yu, Y., Balmant, K., Chen, S., et al. (2016). Preparation of Epidermal Peels and Guard Cell Protoplasts for Cellular,
Electrophysiological, and -omics Assays of Guard Cell Function. Methods Mol. Biol. 1363, 89-121. doi:10.1007/978-1-4939-3115-6_9

Conflict of Interest: Authors JN and CD are employed by Thermo Scientific.

The remaining authors declare that the research was conducted in the absence of any commercial or financial relationships that could be construed as a potential conflict of interest.

Publisher's Note: All claims expressed in this article are solely those of the authors and do not necessarily represent those of their affiliated organizations, or those of the publisher, the editors, and the reviewers. Any product that may be evaluated in this article, or claim that may be made by its manufacturer, is not guaranteed or endorsed by the publisher.

Copyright (c) 2021 David, Kang, Nicklay, Dufresne and Chen. This is an open-access article distributed under the terms of the Creative Commons Attribution License (CC BY). The use, distribution or reproduction in other forums is permitted, provided the original author(s) and the copyright owner(s) are credited and that the original publication in this journal is cited, in accordance with accepted academic practice. No use, distribution or reproduction is permitted which does not comply with these terms. 